\title{
Digital Measurement of the Diameter of the Collagen Fibers by Transmission Electron Microscopy for Diagnosis of Ehlers-Danlos Syndrome-Like in Small Animals
}

\author{
Victor José Vieira Rossetto', Camila Crepaldi Ferranti' ${ }^{2}$, Bruna Fernanda Firmo', Daniela Adachi Miazaki ${ }^{3}$, \\ Daniela Carvalho dos Santos'4, Júlio Lopes Sequeira' \& Cláudia Valéria Seullner Brandão'
}

\begin{abstract}
Background: Ehlers-Danlos-Like Syndrome (EDS) is a rare disease in small animals, whose diagnosis is based on the clinical findings and histopathological examination. The definitive diagnosis may require transmission electron microscopy. Despite this, the ultrastructural changes are poorly described in the literature. The aim of the present study is to describe the ultrastructural findings of collagen fibers and fibroblasts present in the dermis of two animals with EDS, and to evaluate the digital measurement of the diameter of the collagen fibers by transmission electron microscopic images.

Cases: Two animals were evaluated with EDS by transmission electron microscopy. The first animal was an 1-year-old mixed breed female cat, due to spontaneous skin laceration, increased skin elasticity and an extensibility index corresponding to $25 \%$. The second animal evaluated was a 5-month-old Golden Retriever female dog due to articular hypermobility, increased skin elasticity and an extensibility index corresponding to $16.6 \%$. After a skin biopsy of the interscapular and lumbar regions, the samples were fixed in formalin $10 \%$ and glutaraldehyde for, respectively, histopathological examination by HE staining, and transmission electron microscopy. The histopathology of the affected cat revealed collagen fibers shortened and sometimes fragmented. The histopathology of the affected dog revealed disarranged and more eosinophilic staining collagen fibers. The collagen fibers were also of unequal sizes, shortened and slightly undulate. At the transmission electron microscopy of the affected cat was evidenced a greater spacing of the collagen fibers of variable diameters. Further this, the fibroblasts showed elongated nuclei with heterochromatic regions, which was surrounded externally by scant cytoplasm. The cytoplasm showed elongated and discrete organelles. At the transmission electron microscopy of the affected dog was evidenced a greater spacing of the collagen fibers of variable diameters. Further this, fibroblasts exhibited intense cytoplasmic vacuolization with similar appearance to that found in the dying process by autophagy. In addition, the images obtained by transmission electron microscopy were submitted to digital analysis to measure the diameter of the collagen fibers using the software Image J. For this purpose, it was obtained the average of the diameter of 10 collagen fibers in cross-section into four quadrants of $1 \mu \mathrm{m}$ each. The digital analysis of collagen fibers revealed significant alterations in the ultrastructure of collagen. In addition, it was verified cellular changes, such as the large amount of intracytoplasmic vesicles and the small amount of collagen fibers dispersed around the cells.

Discussion: Microscopic abnormalities visualized by HE staining in this present study were compatible with the literature Transmission electron microscopy is fundamental to confirm the suspicion, since it revealed dermal alterations in the ultrastructure of collagen and fibroblasts. These findings indicate possible failure mechanisms of secretion and release of cellular products, as well as collagen. The digital measurement of the diameter of the collagen fibers contributed to the confirmation of the disease, since it was made randomly and reduced the subjectivity inherent of the evaluator. However, there are no studies using this method to allocate a range of significance for the diameter of collagen fibers that may be considered suggestive for the syndrome in small animals.
\end{abstract}

Keywords: asthenia, collagen, Ehlers-Danlos Syndrome.

${ }^{1}$ Department of Veterinary Surgery and Animal Reproduction, School of Veterinary Medicine and Animal Science \& ${ }^{4}$ Department of Structural and Functional Biology, Institute of Biosciences, São Paulo State University (UNESP), Botucatu, SP, Brazil. ${ }^{2}$ Department of Veterinary Surgery and Anesthesiology, Rio Preto Universitary Center (UNIRP), São José do Rio Preto, SP. ${ }^{3}$ Department of Small Animal Surgery, Faculdade de Ensino Superior e Formação Integral (FAEF), Garça, SP. CORRESPONDENCE: V.J.V. Rossetto [vjvrossetto@ hotmail.com]. School of Veterinary Medicine and Animal Science - UNESP. Distrito de Rubião Junior s/n. CEP 18618-970 Botucatu, SP, Brazil. 


\section{INTRODUCTION}

The Ehlers-Danlos-Like Syndrome (EDS) consists of a hereditary and congenital disease that affects connective tissue and the metabolism of collagen and elastic fibers $[4,6,7,9,10,13]$. It is described in dogs and cats and its occurrence is considered rare $[3,7,11,15,16]$. In dogs, the disease may be related to a heterozygous mutation of the COL5A1 gene [3]. In cats, however, the disease may be related to a recessive inheritance associated to breeds [7].

The diagnosis is fundamentally based on history, clinical findings, as increased skin elasticity and suggestive extensibility index (greater than $14.5 \%$ and $19 \%$, respectively in dogs and cats), and on histopathological examination of the skin by Hematoxylin and Eosin (HE) staining [1,6,17]. However, the definitive diagnosis may require transmission electron microscopy, since it can reveal alterations in the ultrastructure of collagen fibers $[3,5-7,9,13,15,17]$.

Despite this, the ultrastructural abnormalities in affected animals are poorly described by the literature that does not provides objective comparative bases, in whom the diagnosis could be grounded. Hence, the aim of the present study is to describe the ultrastructural findings of collagen fibers and fibroblasts present in the dermis of 2 animals with EDS. In addition, it was intended to evaluate the digital measurement of the diameter of the collagen fibers as a possible complementary diagnostic method of Ehlers-Danlos-Like Syndrome in small animals.

\section{CASES}

Case 1. An 1-year-old mixed breed female cat was evaluated due to spontaneous skin laceration, increased skin elasticity and an extensibility index corresponding to $25 \%$.

After a skin biopsy of the interscapular and lumbar regions, the samples were fixed in formalin $10 \%$ and glutaraldehyde for, respectively, histopathological examination by HE staining 1, and transmission electron microscopy.

The histopathology of the affected cat revealed collagen fibers shortened and sometimes fragmented, located in the superficial and middle dermis. No abnormalities were found in the epidermis.

At the transmission electron microscopy of the affected cat was evidenced a greater spacing of the collagen fibers at the longitudinal section of the superficial dermis. The collagen fibers were also fragmented and disposed on a disorganized way. In addition, at the transverse section it was verified disorganized collagen fibers of variable diameters, characterized by some fibers with a very reduced or increased diameter, intercalated to regular ones (Figure 1).

Further this, the fibroblasts in both the epidermis and dermis showed elongated nuclei with heterochromatic regions in small quantity, adhered to its envelope, which was surrounded externally by scant cytoplasm. The cytoplasm showed elongated and discrete organelles, predominantly represented by endoplasmic reticulum, mitochondria and some vesicles. The extracellular matrix near the cells had a moderate amount of collagen fibers arranged irregularly and with twisted appearance (Figure 2).

The digital analysis to measure the diameter of the collagen fibers was performed using the software Image $\mathrm{J}^{2}$. For this purpose, it was obtained the average of the diameter of 10 collagen fibers in cross-section into 4 quadrants of $1 \mu \mathrm{m}$ each. The average diameter of the collagen fibers of the affected cat corresponded to $0.05 \mu \mathrm{m} \pm 0.02 \mu \mathrm{m}$.

Case 2. A 5-month-old Golden Retriever female dog was evaluated due to articular hypermobility, and with historic of several past orthopaedic disorders, including atraumatic rupture of the cranial crucial ligament and craniodorsal hip joint luxation. In addition, the animal presented increased skin elasticity, and the extensibility index value was corresponding to $16.6 \%$.

After a skin biopsy of the interscapular and lumbar regions, the samples were fixed in formalin $10 \%$ and glutaraldehyde for, respectively, histopathological examination by HE staining ${ }^{1}$, and transmission electron microscopy.

The histopathology of the affected dog revealed atrophic epidermis with areas composed of one or two layers of keratinocytes. In the dermis were observed disarranged and more eosinophilic staining collagen fibers of unequal sizes, shortened and slightly undulate. The spaces between the collagen fibers were also increased.

At the transmission electron microscopy of the affected dog was evidenced a greater spacing of the collagen fibers at the longitudinal section of some areas of the superficial dermis. In addition, at the transverse section it was verified a homogeneous pattern of fragmented and disorganized collagen fibers of variable diameters (Figure 1). 


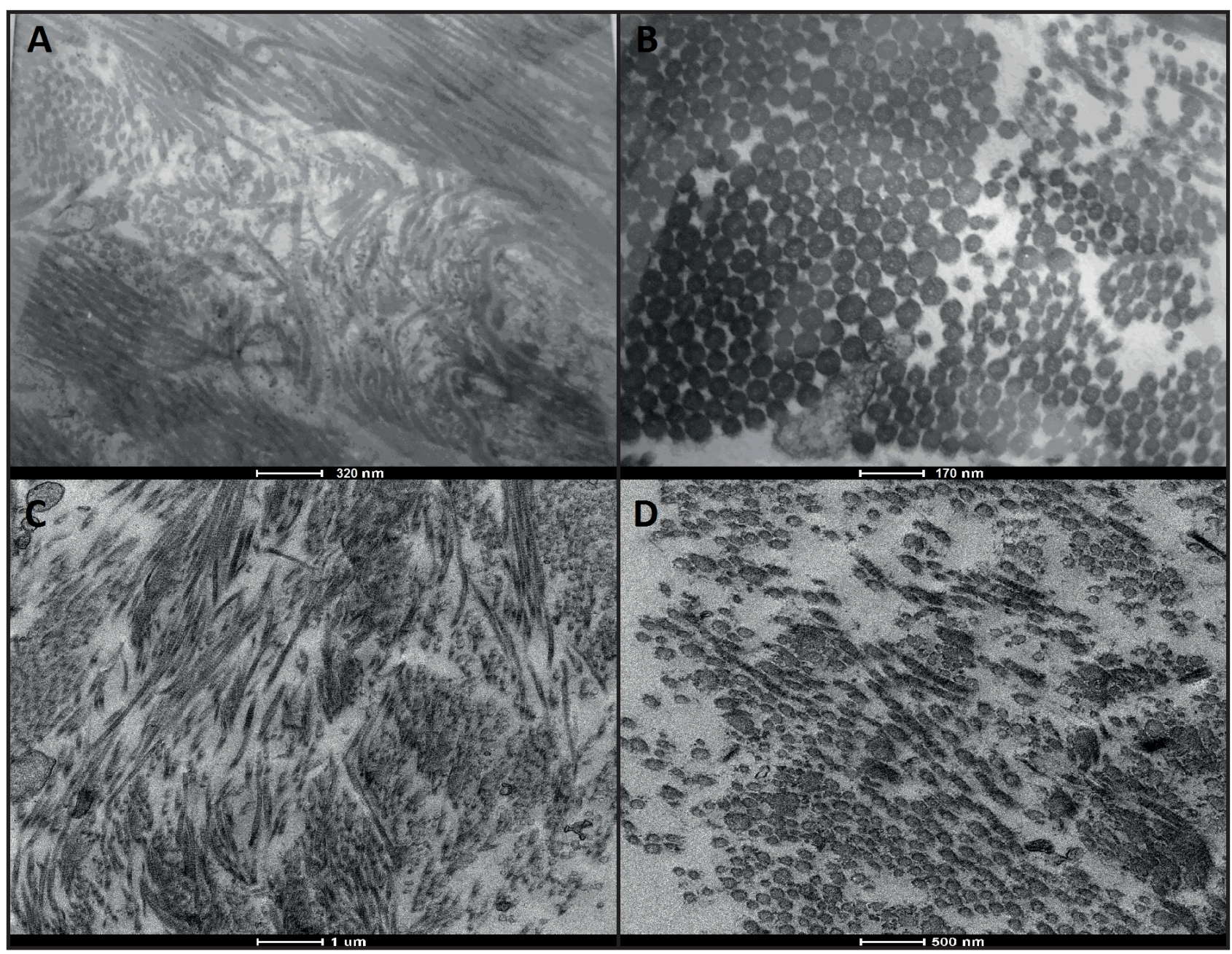

Figure 1. Transmission electron microscopy of the clinically affected animals. A- Longitudinal cut of the clinically affected cat. Note the disorganized and fragmented collagen fibers. B- Transversal cut of the clinically affected cat. Note the important difference in the diameter of the collagen fibers. C- Longitudinal cut of the clinically affected dog. Note the disorganized and fragmented collagen fibers. D- Transversal cut of the clinical affected dog. Note the mild difference in the diameter of the collagen fibers.

Besides that, fibroblasts exhibited intense cytoplasmic vacuolization with similar appearance to that found in the dying process by autophagy, characterized by the presence of numerous vacuoles and vesicles of different sizes and heterogeneous content, and dilated endoplasmic reticulum endosomes. The nuclei showed irregular contours and heterochromatic regions between the euchromatin. The extracellular matrix showed few irregularly arranged collagen fibers of variable sizes and diameters (Figure 2).

The digital analysis to measure the diameter of the collagen fibers was performed using the software Image $\mathrm{J}^{2}$. For this purpose, it was obtained the average of the diameter of 10 collagen fibers in cross-section into four quadrants of $1 \mu \mathrm{m}$ each. The average diameter of the collagen fibers of the affected dog corresponded to $0.08 \mu \mathrm{m} \pm 0.02 \mu \mathrm{m}$.

\section{DISCUSSION}

The general increase in skin elasticity and the index of skin extensibility above the acceptable value, presented in the reported cases, are highly suggestive of EDS and contributed to the disease diagnosis $[2,3,5,7,10,11,17]$.

Additionally to clinical findings, histopathological examination by $\mathrm{HE}$ staining contributes to the disease diagnosis $[5,6,13,17]$. Microscopic abnormalities visualized in this study were compatible with the literature $[5,6,8,13,15,17]$.

Transmission electron microscopy was also fundamental to confirm the suspicion, since it revealed dermal alterations in the ultrastructure of collagen and fibroblasts $[3,5-7,9,13,15,17]$. Both affected cat and the dog had significant cellular changes, such as the large amount of intracytoplasmic vesicles and the small 


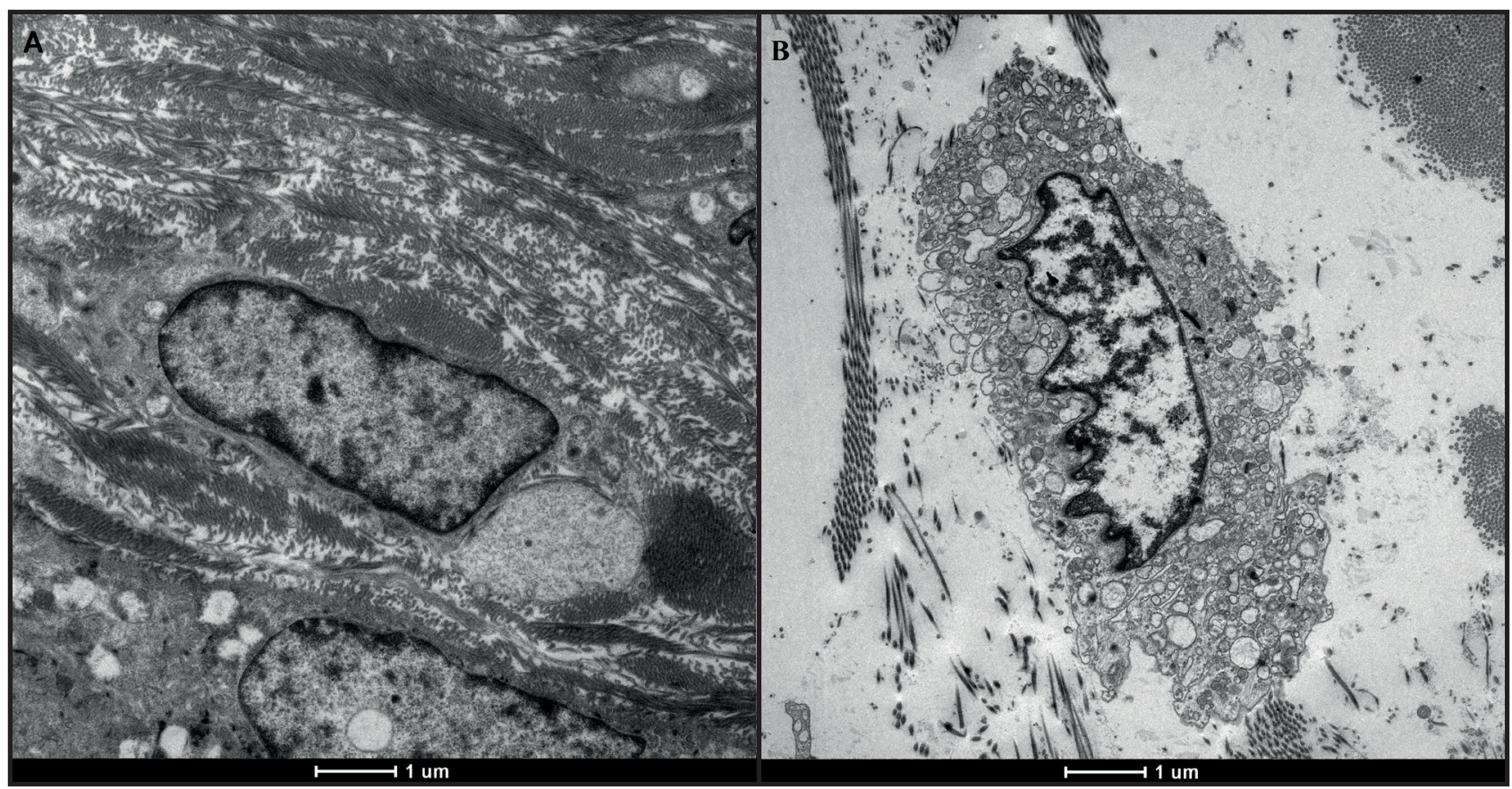

Figure 2. Transmission eletronic microscopy of the clinical affected animals. A- Fibroblast of affected cat. B- Fibroblast of affected dog. Note elongated nuclei with heterochromatic regions and discrete organelles, predominantly represented by endoplasmic reticulum, mitochondria and some vesicles.

amount of collagen fibers dispersed around the cells. These findings indicate possible failure mechanisms of secretion and release of cellular products, as well as collagen [13] and/or cellular autophagy, a process common in stress management of most eukaryotic cells [14]. The poorly defined molecular mechanisms involved in the autophagy process result in the formation of vesicles called autophagosomes, which can engulf entire regions of the cytoplasm, individual organelles, protein aggregates, and invading pathogens [14]. It is believed that the nature of the ESD mechanisms for autophagy in these cases may be involved in the engulfment of protein aggregates.

The digital analysis images enabled the measurement of the diameter of collagen fibers, that tend to be decreased or different sizes in animals with EDS. These findings may be due to deficient synthesis of the enzymes responsible for the processing of the precursor molecules of collagen. The direct result of this is a disorganized aggregation of collagen fibrils that originate collagen bundles of lower or different diameters than the collagen bundles of healthy animals $[3,5,7,12,17]$.
The digital measurement of the diameter of the collagen fibers could contribute to the confirmation of the disease, since it can reduce the subjectivity inherent of the evaluator. However, there are no studies using this method to allocate a range of significance for the diameter of collagen fibers that may be considered suggestive for the syndrome in small animals. Further studies with larger sample size are required for validation of this possible complementary diagnostic method.

\section{MANUFACTURERS}

${ }^{1}$ Sigma-Aldrich Brazil Ltda. São Paulo, SP, Brazil.

${ }^{2}$ National Institutes of Health. Bethesda, MD, USA

Acknowledgements. We thank FAPESP (São Paulo ResearchFoundation) for providing Scientific Initiation Scholarship (number 2017/14098-3). The authors also thank UNIPEX (Unidade de Pesquisa Experimental) - Botucatu.

Ethical approval. This study was approved by the Research Ethics Committee of Botucatu Medical School, UNESP (N'31/2015).

Declaration of interest. The authors report no conflicts of interest. The authors alone are responsible for the content of the paper.

\section{REFERENCES}

1 Andrade S.F., Tostes R.A., Sanches O. \& Melchert A. 2008. Cutaneous Asthenia in Cat. Ciência Animal Brasileira. 9(2): 524-528.

2 Barrera R., Mañe C., Duran E., Vives M.A. \& Zaragoza C. 2004. Ehlers-Danlos syndrome in a dog. Canadian Veterinary Journal. 45(4): 355-356. 
3 Bauer A., Bateman J.F., Lamandé S.R., Hanssen E., Kirejczyk S.G.M., Yee M., Ramiche A., Jagannathan V., Welle M., Leeb T. \& Bateman F.L. 2019. Identification of two independent COL5A1 Variants in dogs with EhlersDanlos Syndrome. Genes. 10(10): 731.

4 De Paepe A. \& Malfait F. 2012. The Ehlers-Danlos syndrome, a disorder with many faces. Clinical Genetics. 82(1): 1-11.

5 Dokzeylul B., Altun E.D., Özdo an T.H., Bozkurt H.H., Arun S.S. \& Or M.E. 2013. Cutaneous asthenia (EhlersDanlos syndrome) in a cat. Turkish Journal of Veterinary and Animal Sciences. 37(1): 245-249.

6 Gross T.L., Ihrke P.J., Walder E.J. \& Affolter V.K. 2005. Degenerative, dysplastic and depositional diseases of dermal connective tissue, In: Gross T.L., Ihrke P.J., Walder E.J. \& Affikter V.K. (Eds). Skin Diseases of the Dog and Cat. Clinical and Histopathologic Diagnosis. 2nd edn. Oxford: Blackwell, pp.373-399.

7 Hansen N., Foster S.F., Burrows A.K., Mackie J. \& Malik R. 2015. Cutaneous asthenia (Ehlers-Danlos-like syndrome) of Burmese cats. Journal of Feline Medicine and Surgery. 17: 954-963.

8 Hargis A.M. 2000. Tegumentar System. In: Carlton W.W., Mcgavin M.D. \& Zachary J.F. (Eds). Thomson's Special Veterinary Pathology. 3rd edn. Oxford: Mosby, pp.500-502.

9 Hermanns-lê T. \& Piérard G.E. 2007. Ultrastructural alterations of elastic fibers and other dermal components in Ehlers-Danlos syndrome of the hypermobile type. American Journal Dermatopathology. 29(4): 370-373.

10 Jaffey J.A., Bullock G., Teplin E., Guo J., Villani N.A., Mhlanga-Mutangadura T., Schnabel R.D., Cohn L.A. \& Johnson G.S. 2019. A homozygous ADAMTS2 nonsense mutation in a Doberman Pinscher dog with Ehlers Danlos syndrome and extreme skin fragility. Animal Genetics. 50(5): 543-545.

11 Medleau L. \& Hnilica K.A. 2006. Congenital Diseases. In: Medleau L. \& Hnilica K.A. (Eds). Small Animal Dermatology: A Color Atlas and Therapeutic Guide. 2nd edn. St. Louis: Elsevier, pp.275-286.

12 Nicholas F.W. 2003. Single-gene Disorders. In: Nicholas F.W. (Ed). Introduction to Veterinary Genetics. 2nd edn. Oxford: Blackwell, pp.75-92.

13 Paciello O., Lamagna F., Lamagna B. \& Papparella S. 2003. Ehlers-Danlos-Like syndrome in 2 dogs: clinical, histologic and ultrastructural findings. Veterinary Clinical Patholology. 32(1): 13-18.

14 Reggiori F., Komatsu M., Finley K. \& Simonsen A. 2012. Selective types of autophagy. International Journal of Cell Biology. 2012: 156272. DOI: 10.1155/2012-156272.

15 Sequeira J.L., Rocha N.S., Bandarra E.P., Figueiredo L.M. \& Eugenio F.R. 1999. Collagen dysplasia (cutaneous asthenia) in a cat. Veterinary Pathology. 36(6): 603-606.

16 M. Szczepanik, M. Goły ski, P. Wilkołek, J. Popiel, A. Śmiech, D. Pomorska \& H. Nowakowski. 2016. EhlersDanlos syndrome (cutaneous asthenia): a report of three cases in cats. Bulletin of the Veterinary Institute in Pulawy. 50: 609-612.

17 Uri M., Verin R., Ressel L., Buckley L. \& McEwan N. 2015. Ehlers-Danlos Syndrome Associated with Fatal Spontaneous Vascular Rupture in a Dog. Journal of Comparative Pathology 152(2-3): 211-216. 